\title{
Metástasis hipofisaria de carcinoma de mama debutando como diabetes insípida
}

\section{Pituitary metastasis from breast carcinoma presenting as diabetes insipidus}

\author{
Julián Castro Castro ${ }^{(1)}$, José Enrique Castro Gómez ${ }^{(2)}$, José Antonio Torre Eiriz ${ }^{(3)}$, Ovidio Fernández Calvo ${ }^{(4)}$ \\ (1) Servicio de Neurocirugía. Complexo hospitalario de Ourense (CHOU). SERGAS. Ourense \\ (2) Servicio de Oncología Radioterápica. Complexo hospitalario de Ourense (CHOU). SERGAS. Ourense \\ (3) Servicio de Otorrinolaringología. Complexo hospitalario de Ourense (CHOU). SERGAS. Ourense \\ (4) Servicio de Oncología Médica. Complexo hospitalario de Ourense (CHOU). SERGAS. Ourense
}

\section{RESUMEN}

Los tumores metastáticos que afectan a la glándula hipofisaria son hallazgos pocos comunes, presentándose en cerca del 1\% de las cirugías hipofisarias. Los autores presentan el caso de una paciente mujer de 46 años que debuta con síntomas de diabetes insípida. Había sido tratada 3 años antes por un carcinoma ductal infiltrante de la mama derecha. Las imágenes de resonancia magnética cerebral mostraron una masa en la silla turca con extensión supraselar. La paciente fue sometida a resección tumoral vía transesfenoidal que demostró metástasis de carcinoma de mama.

PALABRAS CLAVE: carcinoma ductal, diabetes insípida, metástasis, glándula hipofisaria

\section{Introducción}

Las metástasis hipofisarias $(\mathrm{MH})$ son un problema clínico poco frecuente, constituyendo el $0,14-28,1 \%$ de todas las metástasis en las series autópsicas ${ }^{1}$. El empleo de técnicas de imagen más sensibles y el incremento de supervivencia de los pacientes ha provocado el aumento en su frecuencia². Los tumores de mama y de pulmón son las neoplasias primarias que más frecuentemente metastatizan a la hipófisis. Suelen asociarse a una diseminación metastásica marcada, especialmente con afectación ósea.

Aún así, puede ser la primera manifestación de un tumor primario oculto 0 el único lugar de metástasis. Suele presentarse en pacientes en la $6^{\mathrm{a}}$ y $7^{\mathrm{a}}$ décadas, sin clara predominancia de sexos ${ }^{3}$.

Existe una predisposición de las metástasis a afectar a la neurohipófisis y la región infundibular; este fenómeno explicaría la prevalencia de diabetes insípida en estos pacientes; mientras que el fallo adenohipofisario sintomático o las alteraciones de los pares craneales, son menos frecuentes ${ }^{4}$.

\section{Caso clínico}

Presentamos el caso de una mujer de 46 años, tratada 3 años antes en nuestro centro por un carcinoma ductal infiltrante en la mama derecha, estadio IIB. Se había realizado mastectomía y linfadenectomía axilar derechas, recibiendo posteriormente tratamiento radioterápico, quimioterápico y con tamoxifeno.

\begin{abstract}
Metastasic tumors involving the pituitary gland are an uncommon finding and occur in up to $1 \%$ of all pituitary resections. The authors present the case of a 46-year-old woman who presented with symptoms of diabetes insipidus. She had been treated 3 years ago for an invasive ductal carcinoma on the right breast. Magnetic resonance imaging demonstrated a sellar mass with suprasellar extension. The patient underwent a transphenoidal tumor resection that revealed breast carcinoma.

KEY WORDS: ductal carcinoma, diabetes insipidus, metastasis, pituitary gland
\end{abstract}

Refiere un cuadro de 3 meses de evolución de polidipsia, con una ingesta aproximada de 4-5 litros diarios, con preferencia por bebidas frías; acompañado de poliuria y nicturia. Este cuadro se acompaña de astenia, anorexia y pérdida de unos 3-4 Kg.

La exploración neurológica a su ingreso no muestra déficit evidente, perimetría por confrontación normal.

El estudio analítico en plasma y orina es compatible con diabetes insípida. Mostraba niveles de sodio plasmático de 143 mEq/l (rango de 136 a 147 mEq/l), osmolalidad plasmática de 292 mosmol/ $\mathrm{Kg}$ (rango de 275 a $295 \mathrm{mosmo} / \mathrm{Kg}$ ) sodio en orina $29 \mathrm{mmol} / \mathrm{L}$ (rango 54-190 mmol/L) y osmolalidad urinaria 128 (rango 3001300 mosmol/Kg). Se realizó posteriormente test de deprivación hídrica que confirma la sospecha diagnóstica. El resto de estudios hormonales constatan la presencia de un cuadro de panhipopituitarismo. El cuadro clínico de la paciente se atribuye tanto al déficit de hormona antidiurética (ADH) como a la insuficiencia suprarrenal por déficit de ACTH. Ante estos hallazgos se inició tratamiento sustitutivo hormonal con hidrocortisona, levotiroxina y desmopresina.

Se realiza TC craneal con contraste y posteriormente resonancia magnética cerebral demostrando una lesión (ver figura 1) binodular hipofisaria con extensión supraselar y compresión del quiasma; el diámetro vertical de la lesión es de aproximadamente $20 \mathrm{~mm}$, por $15 \mathrm{~mm}$ de diámetro horizontal.

El resto del estudio de extensión no desmuestra otras lesiones sugestivas de metástasis, por lo que se decide tratamiento quirúrgico de la lesión hipofisaria.

Se realizó exéresis quirúrgica transesfenoidal, dejando el resto de lesión supraselar dada su adherencia a las estructuras circun- 
Figura 1. Resonancia magnética cerebral en secuencia T1 con gadolinio; corte axial y sagital. Se demuestra la lesión en forma de "muñeco de nieve" selar y supraselar; realce homogéneo con contraste. Desplaza en sentido craneal el quiasma óptico y rechaza sin invadir los senos cavernosos.
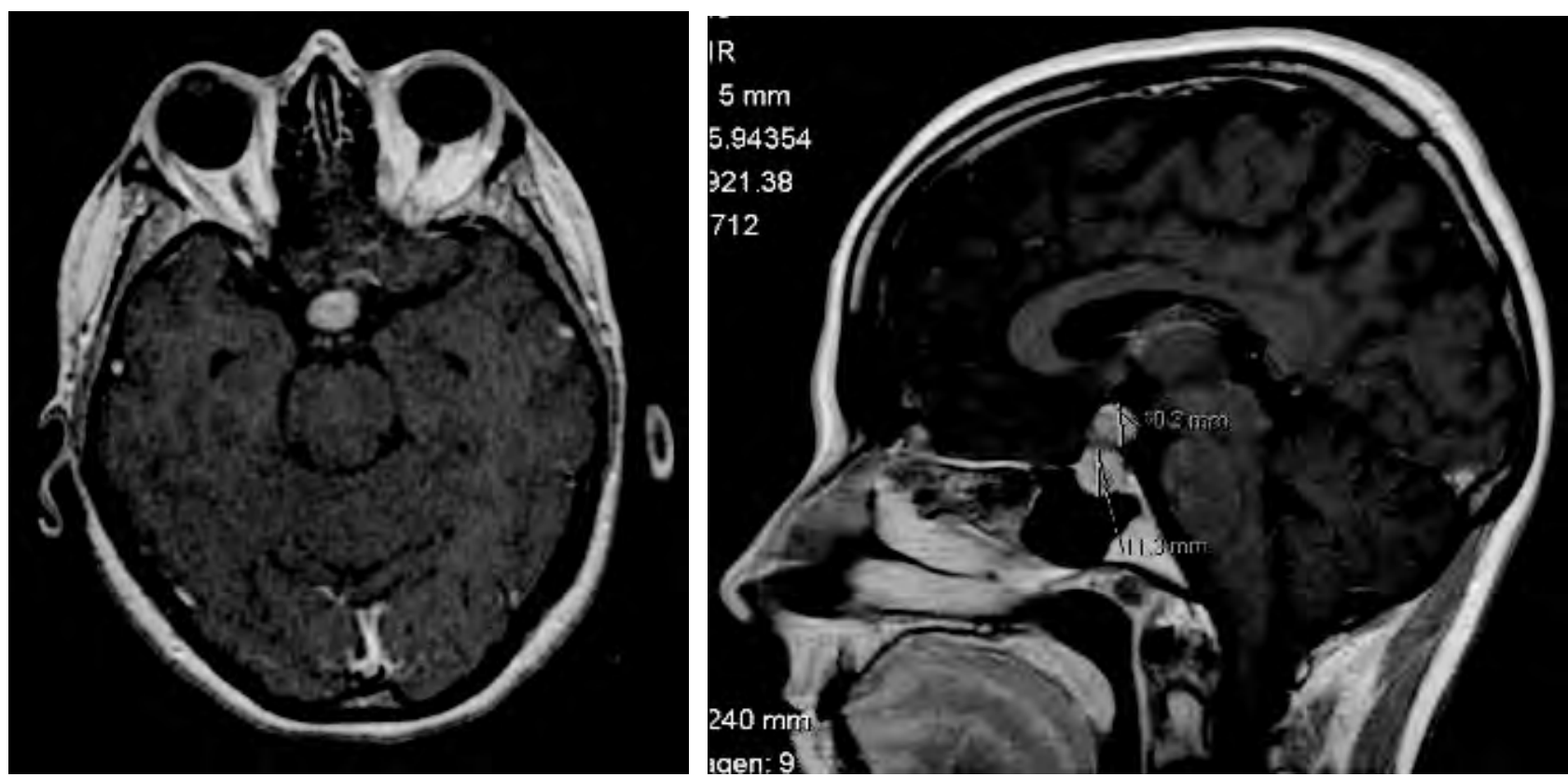

dantes. El resultado de anatomía patológica de esta lesión mostró infiltración por carcinoma ductal de mama. Tras la intervención quirúrgica y dado que el resto de lesión supraselar es la única evidencia de enfermedad metastásica, se decide tratamiento con radioterapia estereotáctica fraccionada.

\section{Discusión}

Las metástasis en la glándula hipofisaria constituyen menos del $1 \%$ de todas las resecciones hipofisarias, sin embargo parecen ocurrir con mayor frecuencia en ciertos tipos tumorales ${ }^{5}$. Las series autópsicas sitúan su incidencia entre el 1 y 3,6\% de los pacientes con tumores malignos, llegando al $28 \%$ si se evalúa la hipófisis y las estructuras adyacentes. El tumor primario más frecuente es el de mama, seguido por el pulmonar. También se han descrito metástasis de origen prostático, renal, tracto gastrointestinal, linfoma, leucemia, carcinoma de tiroides e incluso plasmocitoma ${ }^{2}$. A pesar de la asociación con el cáncer de mama, no parece existir predominancia en el sexo femenin $0^{6}$.

La mayor parte de las MH se sitúan en la hipófisis posterior; afectando sólo en el 13\% de los casos a la hipófisis anterior 0 adenohipófisis. El aporte arterial directo de la neurohipófisis se postula como la causa de este fenómeno; ya que la adenohipófisis recibe su vascularización del sistema portal hipofisario ${ }^{7}$.

La mayor parte de las MH son clínicamente silentes, siendo alrededor del $7 \%$ sintomáticas $^{6}$. Los síntomas más frecuentemente descritos son la diabetes insípida, la oftalmoplejia, cefalea, defectos del campo visual y la disfunción de la hi- pófisis anterior. La alta frecuencia de diabetes insípida se ha relacionado con la predominancia de metástasis afectando al lóbulo posterior de la hipófisis ${ }^{8}$. Dada la invasividad del tipo de tumores que metastatizan a esta región; es frecuente la alteración del campo visual por la invasión supraselar y la oftalmoplejia dolorosa por la invasión del seno cavernoso ${ }^{9}$.

En cuanto al diagnóstico por imagen, existen pocas referencias respecto a los criterios para diferenciar las metástasis hipofisarias de los adenomas. La radiografía simple de cráneo, la tomografía convencional o la angiografía convencional, aportan hallazgos inespecíficos. La tomografía computarizada de alta resolución y la resonancia magnética cerebral son las pruebas más sensibles ${ }^{10}$. Se destacan como características a favor de la metástasis el engrosamiento del tallo hipofisario; la pérdida de la hiperseñal de la glándula hipofisaria normal; isointensidad en secuencias T1 y T2 de resonancia magnética; invasión del seno cavernoso; cambios escleróticos en la silla turca y la morfología bilobulada de la lesión ${ }^{11}$.

Existen múltiples modalidades de tratamiento para las $\mathrm{MH}$. Dada su escasa frecuencia y su presentación en estadios finales de la enfermedad metastásica; no existen estudios significativos comparando los diferentes tratamientos.

La resección quirúrgica se ha asociado a mejoría de los síntomas (agudeza visual, oftalmoplejia) y de la calidad de vida. Sin embargo no se ha demostrado un aumento estadísticamente significativo de la supervivencia global ${ }^{2}$. 
La exéresis quirúrgica suele realizarse vía transesfenoidal, aunque también se ha empleado el abordaje transcraneal. Este tipo de lesiones suelen estar muy vascularizadas e invaden las estructuras adyacentes (senos cavernosos, hipotálamo y aparato óptico), lo que va a impedir en la mayoría de los casos la exéresis completa ${ }^{12}$.

La mayor parte de las series recogen el uso de radioterapia adyuvante, existiendo controversia sobre si debe dirigirse la radiación a la región paraselar o a todo el cráneo. La radiocirugía estereotáctica ha demostrado resultados comparables al empleo de cirugía combinada con radioterapia convencional $^{13}$. El factor limitante para el empleo de la radiocirugía es la dosis de radiación recibida por el aparato óptico.

Diversos agentes quimioterápicos han sido empleados; sin embargo no han sido publicadas las tasas de efectividad en el tratamiento de las metástasis hipofisarias ${ }^{14}$.

El pronóstico de estos pacientes es malo; incluso en pacientes en que la hipófisis es el único lugar de metástasis demostrado; y viene marcado fundamentalmente por la evolución del tumor primario. La supervivencia media es de 6 a 22 meses, sin clara influencia del tratamiento empleado ${ }^{15}$.

\section{Bibliografía}

6. Sioutos P, Yen V, Arbit E. Pituitary gland metastases. Ann Surg Oncol. 1996; 3: 94-99.

7. Morita A, Meyer FB, Laws Jr ER. Symptomatic pituitary metastases. J Neurosurg. 1998; 89: 69-73.

8. Max MB, Deck MD, Rottenberg DA. Pituitary metastasis: incidence in cancer patients and clinical differentiation from pituitary adenoma. Neurology. 1981; 31: 998-1002.

9. Houck WA, Olson KB, Horton J. Clinical features of tumor metastasis to the pituitary. Cancer. 1970; 26: 656-659.

10. Kovacs K: Metastatic cancer of the pituitary gland. Oncology. 1973; 27: 533-542.

11. Teears RJ, Silverman EM: Clinicopathologic review of 88 cases of carcinoma metastatic to the pituitary gland. Cancer. 1975; 36: 216-220.

12. Freda PU, Wardlaw SL, Post KD. Unusual causes of sellar/parasellar masses in a large transsphenoidal surgical series. J Clin Endocrinol Metab. 1996; 81: 3455-3459.

13. Koshimoto $Y$, Maeda M, Naiki H, Nakakuki K, Ishii Y. MR of pituitary metastasis in a patient with diabetes insipidus. Am J Neuroradiol. 1995; 16: 971-974.

14. Kattah JC, Silgals RM, Manz H, et al: Presentation and management of parasellar and suprasellar metastatic mass lesions. J Neurol Neurosurg Psychiatry. 1985; 48: 44-49.

15. Freda PU, Post KD. Differential diagnosis of sellar masses. Endocrinol Metab Clin Am. 1999; 28: 99-100.

16. De Merlier Y, Duprez T, Maiter D, Cosnard G. MR features of pituitary metastases in two patients with central diabetes insipidus. Acta Neurol Belg. 1996; 96: 141-142.

17. Gsponer J, De Tribolet N, Deruaz JP, et al: Diagnosis, treatment, and outcome of pituitary tumors and other abnormal intrasellar masses. Retrospective analysis of 353 patients. Medicine. 1999; 78: 236-269.

18. Petrovich Z, Yu C, Giannotta SL, et al: Survival and pattern of failure in brain metastasis treated with stereotactic gamma knife radiosurgery. J Neurosurg. 2002; 97 (Suppl 5): 499-506.

19. Murata Y, Ogawa Y, Yokoe I, et al: Pituitary stalk metastasis from breast cancer treated with systemic chemotherapy. Oncol Rep. 2003; 10: 1973-1975.

20. Ntyonga-Pono MP, Thomopoulos P, Luton JP. Pituitary metastases. Three cases. Presse Med 1999; 28: 1567-1571. 